\title{
Brief Communication: Caffeic Acid Derivatives and Polymethoxylated Flavonoids from Cat's Whiskers (Orthosiphon stamineus) Form Stable Complexes with SARS-CoV Molecular Targets: An In silico Analysis
}

\author{
Siti Nur Athirah Mohd Kaspi ${ }^{1,2}$, Nisha Govender ${ }^{1 *}$ and Zeti-Azura Mohamed-Hussein ${ }^{1,2}$ \\ ${ }^{1}$ Institute of Systems Biology (INBIOSIS), Universiti Kebangsaan Malaysia, 43600 UKM, Bangi, Selangor, Malaysia \\ ${ }^{2}$ Faculty of Science and Technology, Universiti Kebangsaan Malaysia, 43600 UKM, Bangi, Selangor, Malaysia
}

\begin{abstract}
Cat's whiskers or the 'misai kucing' is an herbal plant native to the Southeast Asian region. The polyphenol enriched leaf extract contains numerous medicinal properties of major pharmaceutical interest. In this study, selected cat's whiskers polyphenols were screened computationally to predict the minimum binding affinities with severe acute respiratory syndrome coronavirus (SARS-CoV) molecular targets. Molecular docking analysis showed that the caffeic acid derivatives and polymethoxylated flavonoids from cat's whiskers bound stably to the binding pocket regions of SARS-CoV molecular targets at -4.2 to -7.1 kcal/mol. Furthermore, these cat's whiskers polyphenol-bound SARS-CoV complexes were held fairly strongly by hydrophobic interactions, hydrogen bonds, and electrostatic interactions at various extents.
\end{abstract}

Keywords: CoV-2, COVID-19, misai kucing, molecular docking, molecular target, polyphenol, SARS- therapeutics

ARTICLE INFO

Article history:

Received: 29 September 2021

Accepted: 06 December 2021

Published: 24 January 2022

DOI: https://doi.org/10.47836/pjtas.45.1.13

E-mail addresses:

a164782@siswa.ukm.edu.my (Siti Nur Athirah Mohd Kaspi) nishag@ukm.edu.my (Nisha Govender)

zeti.hussein@ukm.edu.my (Zeti-Azura Mohamed-Hussein)

* Corresponding author

\section{INTRODUCTION}

The coronavirus disease (COVID-19) first emerged in Wuhan, China, in late 2019. It has been more than a year since the pandemic emerged, and the disease continues to impact the socio-economic lives of the world population. At this time of writing ( $9^{\text {th }}$ November 2021), more than 250 million people have been infected with COVID-19, and up to 5 million deaths 
have been reported globally. Malaysia has reported 2.5 million COVID-19 cases and 29,291 deaths (www.worldometers.info).

COVID-19 is divided into three distinct phases, and each phase represents a unique clinical stage (Gu \& Korteweg, 2007). The asymptomatic stage 1 (about 1-2 days upon infection) begins with the inhalation of airborne droplets of SARS-CoV-2. The virus binds to receptors localized at the epithelial cells in the nasal cavity. Stage 2 (2-5 days upon viral entry) marks the virus propagation at the upper airway and conducting airway, followed by migration heading downwards to the respiratory tract. During this stage, a robust innate immune response is triggered. About $80 \%$ of the COVID-19 infected patients experience mild symptoms when the virus manifestation becomes restricted to the upper and conducting airways ( $\mathrm{Wu}$ \& McGoogan, 2020). In stage 3, massive viral propagation develops into severe disease symptoms as a result of aggravated immune response (cytokine storm) (Tang et al., 2020).

Orthosiphon stamineus Benth. (Clerodendrathus spicatus Thunb.) is wellknown as 'misai kucing' in Malaysia, 'java tea' in Indonesia and 'sen cha' in China (Abdullah et al., 2012; Li et al., 2021). It is one of the most widely grown and used medicinal plants in Asia, southern China, and Australia (Abdullah et al., 2012). The long-term consumption of $O$. stamineus extract (OE) has been shown to offer protection against various diseases (Pandey \& Rizvi, 2009). For thousands of years, the leaves, in their dried and/or fresh form are consumed as a tea to treat multiple ailments such as urinary tract infection (Deipenbrock \& Hensel, 2019), arthritis (Tabana et al., 2016), diabetes mellitus, hypertension, tonsillitis, rheumatism, and menstrual disorders (Cai et al., 2020; Zhong et al., 2012). Bioactive compounds pre-dominantly found in the leaves of $O$. stamineus have been reported to display antiallergic, anti-angiogenic, anti-hypertensive, anti-inflammatory, antioxidant, and diuretic properties (Ashraf et al., 2018, 2020; Muhammad et al., 2011; Tabana et al. 2016). Despite being an important folk medicine, the potential activities of the $O$. stamineus bioactive compounds for COVID-19 management are least reported. Generally, the $O$. stamineus leaves constitute a broad range of bioactive compounds: pentacyclic triterpenes, phenolics, and small sterols. The O. stamineus leaves are especially enriched in polyphenols: up to 0.5 to $0.7 \%$ of the leaf extract contains flavonoids with methoxylated flavones and prenylated flavones, among the majority fraction (Deipenbrock \& Hensel, 2019). In Malaysia, O. stamineus leaf extract has been reported to contain sinensetin, eupatorine, rosmarinic acid, caffeic acid, and gallic acid (EngkuHasmah et al., 2013; Muhammad et al., 2011). In others, chloroform extracts of $O$. staminieus leaves showed high contents of sinensetin and eupatorine flavonoids, whilst the ethanolic leaf extract was characterized with high rosmarinic acid (Tabana et al. 2016).

In this study, the caffeic acid derivatives and polymethoxylated flavonoids from $O$. 
stamineus were docked with SARS-CoV molecular targets at the binding pocket regions, and the minimum binding affinities of the resulting complexes were evaluated. Structural proteins governing key biological functions of the different stages of the SARS-CoV life cycle were identified from Protein Data Bank (PDB). The ligandprotein complexes with good minimum binding affinities were identified and further visualized. Our findings highlight the potentials of $O$. stamineus polyphenols for COVID-19 management strategies such as phytotherapy, preventive therapeutics, and immune health-enhancing and illness relief beverages/supplements.

\section{METHODS}

\section{Data Collection and Target Pre- Processing}

In this study, four different SARS-CoV structural proteins were selected and retrieved from the PDB (www.rcsb.org): membrane protein (PDB ID: 3I6G), main protease (PDB ID: 5RE4), and spike glycoprotein (PDB ID: 6VXX) (PDB ID: $6 \mathrm{VYB})$. The binding pocket regions expressed as $\mathrm{x}, \mathrm{y}$, and $\mathrm{z}$ coordinates in a 3D space were identified using Biovia Discovery Studio 2021 version 4.5. In its native format (.PDB), each protein was manually pre-processed using the AutoDock tools described by Trott and Olson (2010); crystal water molecules were discarded, and hydrogen polar atoms and Kollman charges were added. The edited files were stored in the protein data bank, partial charge (Q), and atom type (T) (PDBQT) format.
The 3D structures of the following $O$. stamineus bioactive compounds (hereafter, designated as ligands) were searched and retrieved from PubChem (pubchem. ncbi.nlm.nih.gov): sinensetin (PubChem Compound ID number [CID]: 145659), gallic acid (PubChem CID: 370), caffeic acid (PubChem CID: 689043), rosmarinic acid (PubChem CID: 5281792), and eupatorine (PubChem CID: 97214). First, the structure data (SDF) format files were converted to PDB format using Open Babel Compiler version 2.3.2. Next, the ligand files were pre-processed using the AutoDock tools (Trott \& Olson, 2010): set the number of torsions and detect the root atoms. Following the edits, all files were saved in the PDBQT format.

\section{Ligand-Receptor Docking}

A configuration file defining the binding pocket regions (grid box) of the receptors was prepared in txt. file. The selected ligands (sinensetin, gallic acid, caffeic acid, rosmarinic acid, and eupatorine) were docked against the binding pocket regions of the receptors using AutoDock Vina 1.1.2. (Trott \& Olson, 2010). All docking runs were initiated at Command Prompt (Windows operating system). The output log files were evaluated, and the best-docked conformations were selected based on binding energy ( $\mathrm{kcal} / \mathrm{mol})$, cluster RMSD value, number of stabilizing interactions such as hydrogen bonds, and other weak interactions. The 3D complex structure and interactions were visualized using the Biovia Discovery Studio version 4.5. 


\section{RESULTS AND DISCUSSION}

The coronavirus disease-2019 (COVID-19) is caused by severe acute respiratory syndrome coronavirus 2 (SARS-CoV-2). The SARS-CoV-2 pathogenesis mechanism is governed by structural proteins (surface spike protein, membrane protein, envelope protein, and main protease-replicase polyproteins) and a few non-structural proteins (nsp6, nsp8, and nsp10) (Walls et al., 2020). The global outbreak is one of the most challenging pandemics ever experienced, and effective drugs are yet to avail (November 2021). However, numerous research efforts, especially promising and high-throughput computational biology approaches for plant-based therapeutics, have emerged through molecular docking, modeling, and dynamic simulation studies (Skariyachan et al., 2020).

The O. stamineus is a traditional medicinal herbal plant with rich bioactive compounds. Extensively concentrated in the leaves, there are a large number of different types of bioactive compounds identified in O. stamineus (Li et al., 2021; Tezuka et al., 2000). As such, the total phenolic content of $O$. stamineus leaf $50 \%$ ethanol extract was $385 \mu \mathrm{g} / \mathrm{ml}$, and the total flavonoid content, $57 \mu \mathrm{g} / \mathrm{ml}$ (Tabana et al., 2016). Rosmarinic acid is the main constituent of O. stamineus leaves. Previous studies have reported that $O$. stamineus leaves contain two different polyphenols in abundance: polymethoxylated flavonoids and caffeic acid derivatives (Engku-Hasmah et al., 2013; Muhammad et al., 2011; Tabana et al., 2016). This study selected the most abundantly occurring polyphenols in the O. stamineus leaves as ligands. Sinestine and eupatorine are polymethoxylated flavonoids, while rosmarinic acid, gallic acid, and caffeic acid belong to the caffeic acid derivatives group (Olah et al., 2003).

Four different structural SARS-CoVrelated proteins were selected, namely the membrane protein (PDB ID: 3I6G), main proteases (PDB ID: 5RE4), and spike glycoproteins (PDB ID: 6VXX and 6VYB). The biological roles of the selected proteins are described as follows: $3 \mathrm{I} 6 \mathrm{G}$ is a SARS-CoV M protein complexed with HLA-A*0201 obtained from X-ray diffraction method; 5RE4 is a crystal structure of SARS-CoV-2 main protease complexed with Z1129283193 obtained by X-ray diffraction method; 6VXX is a closed state model of SARS-CoV-2 spike glycoprotein obtained via electron microscopy, and 6VYB, an open state of SARS-CoV-2 spike ectodomain structure obtained through electron microscopy (www.rcsb.org).

The minimum binding affinities for all possible combinations of ligand (sinensetin, gallic acid, caffeic acid, rosmarinic acid, and eupatorine)-protein complexes were measured at root mean square deviation $(\mathrm{RSMD})=0$. For complexes formed between the ligands and the $3 \mathrm{I} 6 \mathrm{G}$, the binding affinity ranged from -5.3 to $-7.1 \mathrm{kcal} / \mathrm{mol}$ (Table 1). A various number of hydrogen bonds, hydrophobic, and electrostatic interactions were observed at the receptor residue (RR) of all 3I6G-bound complexes except the 3I6G-gallic acid complex (absence of 
hydrophobic interaction) (Table 2). The rosmarinic acid-3I6G complex showed the highest binding affinity at $-7.1 \mathrm{kcal} / \mathrm{mol}$, followed by sinensetin-3I6G complex at $-6.6 \mathrm{kcal} / \mathrm{mol}$ (Table 1 ). In contrast, the number of interactions was higher in the sinensetin-3I6G complex as compared to the rosmarinic acid-3I6G complex. There were three hydrogen bonds (RR: ARG9 7, TYR123, HIS114), three hydrophobic interactions (RR: VAL152, TRP147, ALA150), and one electrostatic interaction (ASP77) in the rosmarinic acid-3I6G complex. In comparison, the sinensetin3I6G complex showed three hydrogen bonds (RR: TYR7, GLU63, LYS66), six hydrophobic interactions (RR: TYR7, TYR159, PHE9, HIS70, TRP167, LYS66), and a single electrostatic interaction (RR: LYS66) (Figure 1A) (Table 2). On the other hand, 3I6G-gallic acid showed the lowest binding affinity at $5.3 \mathrm{kcal} / \mathrm{mol}$ with a single hydrogen bond (RR: TRP14) and electrostatic interaction (RR: ASP77) (Tables 1 and 2).

The minimum binding affinities of ligands bound to 5RE4 ranged from -4.9 to $-6.7 \mathrm{kcal} / \mathrm{mol}$. The rosmarinic acid-5RE4 complex shows the least minimum binding affinity at $-6.7 \mathrm{kcal} / \mathrm{mol}$, followed by the eupatorine-5RE4 and sinensetin-5RE4 complexes at $-5.7 \mathrm{kcal} / \mathrm{mol}$ and $-5.6 \mathrm{kcal} /$ mol, respectively (Table 1). Although the rosmarinic acid-5RE4 complex showed no presence of electrostatic interactions, a total of nine hydrogen bonds (RR: ARG76, LEU75, PHE66, ASP92, THR93, HIS64, LEU67, LEU75, VAL77) along two hydrophobic interactions (RR: ARG76, VAL73) were observed (Figure 1B). The eupatorine-5RE4 complex showed the presence of a hydrogen bond (RR: VAL77), hydrophobic interaction (RR: ARG76), and electrostatic (RR: ARG76) interaction each. Besides the rosmarinic acid-5RE4 complex, both the gallic acid-5RE4 and caffeic acid5RE4 complexes showed an absence of electrostatic interaction (Table 2).

For the ligand-6VXX complexes, the minimum binding affinities range at -4.3 to $-5.6 \mathrm{kcal} / \mathrm{mol}$ (Table 1 ), and electrostatic interactions were consistently absent in all complexes (Table 2). The eupatorine-6VXX complex showed the least minimum binding affinity at $-5.6 \mathrm{kcal} / \mathrm{mol}$ with three hydrogen bonds (RR: PHE86, ASN87, ASN234)

Table 1

Molecular docking analysis of Orthosiphon stamineus polyphenols and SARS-CoV molecular targets (proteins) performed using the Autodock Vina software

\begin{tabular}{lcccc}
\hline & 3I6G & 5RE4 & 6 VXX & 6VYB \\
\hline Sinensetin & -6.6 & -5.6 & -4.9 & -4.8 \\
Gallic acid & -5.3 & -4.9 & -4.7 & -4.5 \\
Caffeic acid & -5.4 & -5.0 & -4.3 & -4.2 \\
Rosmarinic acid & -7.1 & -6.7 & -5.5 & -6.0 \\
Eupatorine & -6.5 & -5.7 & -5.6 & -5.6 \\
\hline
\end{tabular}

Note. The first row denotes receptor ID (Protein Data Bank), and the first column represents polyphenols. All numerical values indicate the minimum binding affinities expressed in $\mathrm{kcal} / \mathrm{mol}$ 
Table 2

The interactions between the Orthosiphon stamineus polyphenol and receptor residue

\begin{tabular}{|c|c|c|c|}
\hline $\begin{array}{l}\text { Receptor-ligand } \\
\text { complex }\end{array}$ & Hydrogen bond & Hydrophobic interaction & $\begin{array}{c}\text { Electrostatic } \\
\text { interaction }\end{array}$ \\
\hline 3I6G-sinensetin & TYR7, GLU63, LYS66 & $\begin{array}{l}\text { TYR7, TYR159, PHE9, } \\
\text { HIS70, TRP167, LYS66 }\end{array}$ & LYS66 \\
\hline 3I6G-gallic acid & TRP14 & - & ASP77 \\
\hline 3I6G-caffeic acid & LYS66, ARG97, HIS114 & TYR15 & LYS66 \\
\hline 3I6G-rosmarinic & ARG97, TYR123, HIS114 & VAL152, TRP147, ALA150 & ASP77 \\
\hline 3I6G-eupatorine & TYR7, LYS66 & $\begin{array}{c}\text { TYR7, TYR159, LEU156, } \\
\text { PHE9, LYS66 }\end{array}$ & LYS66 \\
\hline 5RE4-sinensetin & PHE66, LEU75 & $\begin{array}{c}\text { VAL73, LEU75, LEU67, } \\
\text { ARG76 }\end{array}$ & $\begin{array}{l}\text { ARG76, } \\
\text { ASP92 }\end{array}$ \\
\hline 5RE4-gallic acid & GLN74, PHE66, VAL77 & ARG76, VAL77 & - \\
\hline 5RE4-caffeic acid & GLN74, PHE66, HIS64, VAL77 & ARG76, VAL77 & - \\
\hline 5RE4-rosmarinic & $\begin{array}{c}\text { ARG76, LEU75, PHE66, ASP92, } \\
\text { THR93, HIS64, LEU67, LEU75, } \\
\text { VAL77 }\end{array}$ & ARG76, VAL73 & - \\
\hline 5RE4-eupatorine & VAL77, & ARG76 & ARG76 \\
\hline 6VXX-sinensetin & THR236, ILE233 & ARG237, THR236 & - \\
\hline 6VXX-gallic acid & ASN196, ILE237, ASN234, ILE235 & - & - \\
\hline 6VXX-caffeic acid & - & - & - \\
\hline 6VXX-rosmarinic & ASP88, ASN196, THR236 & - & - \\
\hline 6VXX-eupatorine & PHE86, ASN87, ASN234, & THR236, PHE86 & - \\
\hline 6VYB-sinensetin & THR236, ASN234 & THR236, ARG237 & - \\
\hline 6VYB-gallic acid & THR108, LYS113, GLY232 & ILE233 & - \\
\hline 6VYB-caffeic acid & ILE235 & - & - \\
\hline 6VYB-rosmarinic & THR236, ILE235 & - & - \\
\hline 6VYB-eupatorine & - & - & - \\
\hline
\end{tabular}

and two hydrophobic interactions (RR: THR236, PHE86) (Figure 1C). The caffeic acid-6VXX complex showed the lowest binding affinity at $-4.3 \mathrm{kcal} / \mathrm{mol}$ with no interactions (Table 1).

The minimum binding affinities for ligand-6VYB ranged from -4.2 to -6.0 $\mathrm{kcal} / \mathrm{mol}$. The rosmarinic acid-6VYB complex showed the least minimum binding affinity at $-6.0 \mathrm{kcal} / \mathrm{mol}$, followed by the eupatorine-6VYB complex at $-5.6 \mathrm{kcal} /$ mol. Caffeic acid-6VYB complex showed the least minimum binding affinity $(-4.2$ $\mathrm{kcal} / \mathrm{mol}$ ) among all the ligand-6VYB complexes (Table 1). All the ligand-6VYB complexes had no electrostatic interaction. Interestingly, the sinensetin-6VYB complex showed a relatively lower minimum binding affinity $(-4.8 \mathrm{kcal} / \mathrm{mol})$ than the rosmarinic acid-6VYB complex $(-6.0 \mathrm{kcal} / \mathrm{mol})$ and the number of interactions displayed a vice versa trend. The rosmarinic acid-6VYB complex showed two hydrogen bonds (RR: THR236, ILE235) only, while the 


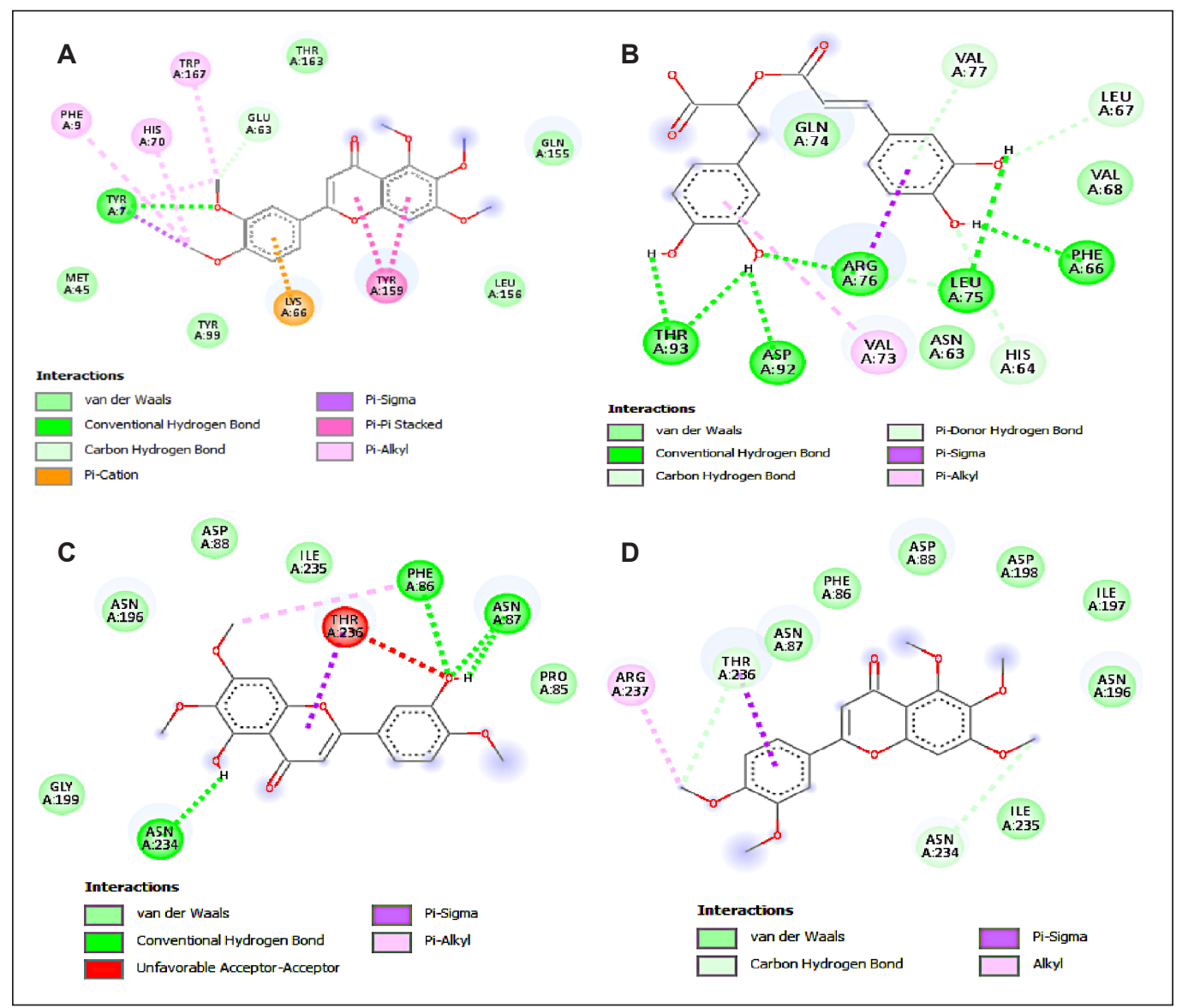

Figure 1. Interactions between the Orthosiphon stamineus polyphenols and SARS-CoV receptors. A) 3I6G-sinensetin complex; B) 5RE4-rosmarinic acid complex; C) 6VXX-eupatorine complex, and D) 6VYBsinensetin complex. The best conformation complexes are selected based on the minimum binding affinity values and number of interactions at RMSD $=0$. All 2- dimension $O$. stamineus polyphenol-bound SARS$\mathrm{CoV}$ receptors complexes contain nodes, represent the receptor residue (RR), and dotted lines represent the interactions between ligand atom and RR

sinensetin-6VYB complex showed a greater number of interactions with two hydrogen bonds (RR: THR236, ASN234) and two hydrophobic interactions (RR: THR236, ARG237) (Figure 1D) (Table 2).

Plant-based natural products have been used to treat various diseases for a long time. These natural resources are mainly derived from beneficial traditional plant medicines. In time of the COVID-19 pandemic, the use of traditional medicinal plants as alternative medicine has been overwhelming, especially among Asian countries. Both China and the Indian government have recommended polyherbal decoctions for COVID-19 prevention and management: Qingfei Paidu, Huashi Baide, Xuanfei Badu, Toujie Quwen granules, Jinhua Qinggan granules (traditional Chinese medicine), Kabasura Kudineer 
(traditional Siddha medicine). In Thailand, Andrographis paniculata (local name, 'hempedu bumi') is used for mild COVID-19 infection, whilst other herbal plants such as the lemon balm (Melissa officinalis L.), skullcap (Scutellaria lateriflora), Siberian ginseng (Eleutherococcos senticosus) are also widely employed throughout the world. As plant-based medicines pose minimal side effects compared to steroidal drugs, the ethnopharmacological relevance of traditional herbal plants for COVID-19 can be explored using extensive computational approaches, bioinformatics, and cheminformatics. In this study, five different polyphenols enriched in $O$. stamineus leaves (the sinensetin, caffeic acid, gallic acid, rosmarinic acid, and eupatorine) showed structural interactions with SARS-CoV proteins. In terms of enzymes encoding pathogenesis, the SARS$\mathrm{CoV}$ is similar to SARS-CoV-2 (Lan et al., 2020). The structural insights of the potential interactions between the $O$. staminineus polyphenols and SARS-CoV proteins require further investigations to confirm and fully validate the inhibitory properties of $O$. staminineus polyphenols against SARS-CoV proteins. Other molecular dynamic simulation studies, drug-likeness analysis, and bioassay studies are necessary to identify the inhibitory and druggability roles of $O$. staminineus polyphenols against SARS-CoV-2 of COVID-19. The use of $O$. stamineus to treat multiple ailments could be stretched to viral diseases associated with SARS-CoV, such as the COVID-19.

\section{CONCLUSION}

The in silico analysis of Orthosiphon stamineus polyphenols against SARS-CoV molecular targets shows good interaction at a minimum binding affinity range of -4.2 to $-7.1 \mathrm{kcal} / \mathrm{mol}$ coupled with numerous hydrogen bonds and hydrophobic interactions. The $O$. stamineus polyphenols are lucrative alternatives to synthetic drugs as they are comparably cheap and may pose fewer side effects in anti-viral treatments. With further research that complements the present preliminary findings, $O$. staminineus polyphenols could potentially be utilized for COVID-19 therapeutics development.

\section{ACKNOWLEDGEMENTS}

The first author would like to thank the Institute of Systems Biology (INBIOSIS), Universiti Kebangsaan Malaysia, Bangi, Selangor, Malaysia, for the internship training.

\section{REFERENCES}

Abdullah, S., Shaari, A. R., \& Azimi, A. (2012). Effect of drying methods on metabolites composition of misai kucing (Orthosiphon stamineus) leaves. APCBEE Procedia, 2, 178-182. https://doi. org/10.1016/j.apcbee.2012.06.032

Ashraf, K., Halim, H., Lim, S. M., Ramasamy, K., \& Sultan, S. (2020). In vitro antioxidant, antimicrobial and antiproliferative studies of four different extrscts of Orthosiphon stamineus, Gynura procumbens and Ficus deltoidei. Saudi Journal of Biological Sciences, 21(1), 417-432. https://doi.org/10.1016/j.sjbs.2019.11.003

Ashraf, K., Sultan, S., \& Adam, A. (2018). Orthosiphon stamineus Benth. is an outstanding food medicine: Review of phytochemical and 
pharmacological activities. Journal of Pharmacy and Bioallied Sciences, 10(3), 109-118. https:// doi.org/10.4103/jpbs.JPBS_253_17

Cai, X., Zhang, L., Chen, X., Zhang, H., Xue, H., Lu, Y., Tang, J., \& Lu, Y. (2020). Orthosiphon stamineus and rosmarinic acid reduce heat stress in laying hens. Livestock Science, 240, 104124. https://doi.org/10.1016/j.livsci.2020.104124

Deipenbrock, M., \& Hensel, A. (2019). Polymethoxylated flavones from Orthosiphon stamineus leaves as antiadhesive compounds against uropathogenic E. coli. Fitoterapia, 139, 104387. https://doi.org/10.1016/j. fitote. 2019.104387

Engku-Hasmah, E. A., Ahmad Tarmizi, S., Noor Ismawaty, N., \& Abdul Ghani, O. (2013). Total phenolic, total flavonoid and rosmarinic acid contents in Orthosiphon stamineus (Misai Kucing) in relation to storage conditions. Acta Horticulturae, 1012, 837-842. https://doi. org/10.17660/ActaHortic.2013.1012.113

Gu, J., \& Korteweg, C. (2007). Pathology and pathogenesis of severe acute respiratory syndrome. The American Journal of Pathology, 170(4), 1136-1147. https://doi.org/10.2353/ ajpath.2007.061088

Lan, J., Ge, J., Yu, J., Shan, S., Zhou, H., Fan, S. (2020). Crystal structure of the 2019-nCoV spike receptor-binding domain bound with ACE2 receptor. https://www.biorxiv.org/content/10.11 01/2020.02.19.956235v1.full.pdf

Li, Z., Qu, B., Zhou, L., Chen, H., Wang, J., Zhang, W., \& Chen, C. (2021). A new strategy to investigate the efficacy markers underlying the medicinal potentials of Orthosiphon stamineus Benth. Frontiers in Pharmacology, 12, 748684. https://doi.org/10.3389/fphar.2021.748684

Muhammad, H., Gomes-Carneiro, M. R., Poa, K. S., De-Oliveira, A. C. A. X., Afzan, A., Sulaiman, S. A., Ismail, Z., \& Paumgartten, F. J. R. (2011). Evaluation of the genotoxicity of
Orthosiphon stamineus aqueous extract. Journal of Ethnopharmacology, 133(2), 647-653.

Olah, N. K., Radu, L., Mogosan, C., Hanganu, D., \& Gocan, S. (2003). Phytochemical and pharmacological studies on Orthosiphon stamineus Benth. (Lamiaceae) hydroalcoholic extracts. Journal of Pharmaceutical and Biomedical Analysis, 33(1), 117-123. https:// doi.org/10.1016/s0731-7085(03)00227-9

Pandey, K. B., \& Rizvi, S. I. (2009). Plant polyphenols as dietary antioxidants in human health and disease. Oxidative Medicine and Cellular Longevity, 2, 897484. https://doi.org/10.4161/ oxim.2.5.9498

Skariyachan, S., Gopal, D., Chakrabarti, S., Kempanna, P., Uttarkar, A., Muddebihalkar, A. G., \& Niranjan, V. (2020). Structural and molecular basis of the interaction mechanism of selected drugs towards multiple targets of SARS-CoV-2 by molecular docking and dynamic simulation studies-deciphering the scope of repurposed drugs. Computational Biology Medicine, 126, 104054. https://doi.org/10.1016/j. compbiomed.2020.104054

Tabana, Y. M., Al-Suede, F. S., Ahamed, M. B., Dahham, S. S., Hassan, L. E., Khalilpour, S., Taleb-Agha, M., Sandai, D., Majid, A.S.A., \& Majid, A. M. S. A. (2016). Cat's whiskers (Orthosiphon stamineus) tea modulates arthritis pathogenesis via the angiogenesis and inflammatory cascade. BMC Complementary and Alternative Medicine, 16(1), 480. https:// doi:10.1186/s12906- 016-1467-4

Tang, Y., Liu, J., Zhang, D., Xu, Z., Ji, J., \& Wen, C. (2020). Cytokine storm in COVID-19: The current evidence and treatment strategies. Frontiers in Immunology, 11, 1708. https://doi. org/10.3389/fimmu.2020.01708

Tezuka, Y., Stampoulis, P., Banskota, A. H., Awale, S., Tran, K. Q., Saiki, I., \& Kadota, S. (2000). Constituents of the Vietnamese medicinal 
plant Orthosiphon stamineus. Chemical and Pharmaceutical Bulletin, 48(110),1711-1719.

Trott, O., \& Olson, A. J. (2010). AutoDock Vina: Improving the speed and accuracy of docking with new scoring function, efficient optimization and multithreading. Journal of Comptutational Chemistry, 31(2), 455-461. https://doi. org/10.1002/jcc. 21334

Walls, C., Park., Y., Tortorici, M. A., Wall, A., McGuire, A. T., \& Veesler, D. (2020). Structure, function and antigenicity of the SARS-CoV-2 spike glycoprotein. Cell, 181(2), 281-292. https://doi.org/10.1016/j.cell.2020.02.058
Wu, Z., \& McGoogan, J. M. (2020). Characteristics of and important lessons from the coronavirus disease 2019 (COVID-19) outbreak in China: Summary of a report of 72314 cases from the Chinese Center for Disease Control and Prevention. JAMA - Journal of the American Medical Association, 323(13), 1239-1242. https://doi.org/10.1001/jama.2020.2648

Zhong, Y. S., Yu, C. H., Ying, H. Z., Wang, Z. Y., \& Cai, H. F. (2012). Prophylactic effects of Orthisiphon stamineus Benth. extracts on experimental induction of calcium oxalate nephrolithiasis in rats. Journal of Ethnopharmacology, 144(3), 761-767. 